\title{
Factores implicados para realizar o no realizar tesis en estudiantes de psicología
}

\section{Factors involved in the Decision of whether to do or not a Thesis in Psychology Students}

Jesús J. Aiquipa*®i Universidad Nacional Mayor de San Marcos, Lima, Perú. ORCID: https://orcid.org/0000-0002-1982-6908

Cinthia M. Ramos@Universidad Inca Garcilaso de la Vega, Lima, Perú. ORCID: https://orcid.org/0000-0002-1170-9715

Rosmery Curay Universidad Inca Garcilaso de la Vega, Lima, Perú. ORCID: https://orcid.org/0000-0002-8462-6793

Leidy L. Guizado@Universidad Inca Garcilaso de la Vega, Lima, Perú. ORCID: https://orcid.org/0000-0002-6082-8024

Recibido 04-09-17 Revisado 21-09-17 Aprobado 27-11-17 En línea 06-12-17

*Correspondencia

Email: jesus.aiquipat@gmail.com
Citar como:

Aiquipa, J.J., Ramos, C.M., Curay, R., \& Guizado L.L. (2018). Factores implicados para realizar o no realizar tesis en estudiantes de psicología. Propósitos y Representaciones, 6(1), 21-82. doi: http:// dx.doi.org/10.20511/pyr2018.v6n1.180

(c) Universidad San Ignacio de Loyola, Vicerrectorado de Investigación, 2018.

(cc)BY-NC-ND Este artículo se distribuye bajo licencia CC BY-NC-ND 4.0 Internacional (http://creativecommons.org/licenses/by-nc-nd/4.0/). 


\section{Resumen}

A pesar de la necesidad de investigaciones científicas en Psicología para dar respuesta a los diferentes problemas psicosociales del país (violencia familiar y corrupción, por ejemplo), los estudios en esta área son aún insuficientes. Una de las formas de incentivar las investigaciones es a través de la realización de tesis. Sin embargo, muchos estudiantes optan por otro tipo de trabajos para tal fin. El objetivo del estudio es identificar, describir y explicar aquellos factores que influyen en la decisión de realizar o no realizar tesis para obtener el título profesional en estudiantes de pregrado de la carrera de Psicología. Se empleó una metodología cualitativa y el diseño Teoría Fundamentada. Se realizaron 7 entrevistas individuales y 2 grupos focales a un total de 28 estudiantes universitarios (20 mujeres y 8 varones), residentes en la ciudad de Lima, de 2 universidades nacionales y 3 particulares, que cursaban los últimos ciclos de la carrera de Psicología. Los resultados sugieren que existen factores psicológicos, circunstanciales, familiares, relacionados a la universidad, sociales y culturales cuya compleja interacción influye en los procesos psicológicos del estudiante para tomar la decisión de realizar o no realizar una tesis, representando el sistema de creencias del estudiante respecto de sí mismo y en relación con la actividad de investigación un factor psicológico central.

Palabras clave: Psicología, tesis, universidad, sistema de creencias.

\section{Summary}

Despite the need for scientific research in psychology to solve the different psychosocial problems of the country (family violence and corruption, for example), studies in this area are not still enough. One of the ways to encourage research is by doing a thesis. However, many students opt for other types of jobs for that purpose. The objective of the study is to identify, describe and explain those factors that influence the undergraduate Psychology students' decision to do or not a thesis in order to obtain the 
professional degree. A qualitative methodology and the Grounded Theory design were used. We conducted 7 individual interviews and 2 focus groups of a total of 28 university students (20 women and 8 men), living in the city of Lima, 2 of them from public universities and 3 of them from private universities and who were in the last semesters of the Psychology specialty. The results suggest that there are psychological, circumstantial, family, university-related, social and cultural factors, complex interaction of which affects the psychological processes of the student to decide to do or not a thesis, representing the student's belief system in itself and in relation to the research activity, a main psychological factor.

Keywords: Psychology, thesis, university, belief system. 


\section{Introducción}

Se entiende por tesis el producto de un proceso de investigación científica, empírica y/o teórica, sobre un fenómeno particular, el cual ha sido adquirido durante la elaboración de un trabajo de acumulación de información, análisis y desarrollo de conocimiento durante un considerado periodo de tiempo (Montemayor-Borsinger, 2005); implica un trabajo riguroso y ordenado en su forma y contenido, original y creativo (Sabino, 1998) y tiene por finalidad la obtención de un grado académico o título universitario (Arias, 2006).

Según el último estudio registrado en Perú, el II Censo Nacional Universitario 2010 (Instituto Nacional de Estadística e Informática [INEI], 2011), efectuado simultáneamente en 100 Universidades del país (35 públicas y 65 privadas), reportó que, de un total de 20,642 estudiantes de universidades públicas, el $45 \%$ se tituló mediante la realización de tesis, mientras que $55 \%$ obtuvo el título por otras modalidades (experiencia profesional, examen, curso de actualización y/o clases magistrales). Asimismo, de un total de 24,601 alumnos de postgrado de universidades privadas, el $40 \%$ obtuvo el título realizando una tesis, mientras que el $60 \%$ se tituló por medio de otras modalidades (INEI, 2011).

En este contexto, es oportuno mencionar que la Ley Universitaria (Ley No 30220, 2014), en su Capítulo V de Organización Académica, Artículo 45. Obtención de grados y títulos, señala que la obtención de grados y títulos se realiza de acuerdo a las exigencias académicas que cada universidad establezca en sus respectivas normas internas, siendo requisito para la obtención del grado de bachiller, entre otros puntos, la aprobación de un trabajo de investigación, y para la obtención del Título Profesional se requiere la aprobación de una tesis o trabajo de suficiencia profesional. Si bien, la ley señala que las universidades acreditadas pueden establecer modalidades adicionales a las referidas, el hacer un trabajo de investigación es la prioridad y garantiza un país a la vanguardia de la ciencia, tecnología e innovación, 
pues sus profesionales se encuentran capacitados para realizar estudios y contribuir con el crecimiento económico, social y cultural del país.

Por otro lado, en el ámbito peruano, son muchos los problemas de la salud mental que generan perjuicios en los individuos y familias, además de las consecuencias económicas y sociales que ello implica (Defensoría del Pueblo, 2008). Algunos de estos problemas son la violencia familiar, feminicidio, hostigamiento escolar, acoso sexual en los medios de transporte público, altos niveles de corrupción, conductas autolesivas y suicidas, hábitos de salud inadecuados relacionados a problemas físicos y accidentes. Por ello, es una necesidad realizar investigaciones en el ámbito psicológico, en todas sus áreas, a fin de contribuir con el conocimiento científico sobre sus efectos, percepciones y/o factores de riesgos que facilitan la incidencia de tales problemas, de tal manera que se cuente con una base empírica para poder implementar y ejecutar propuestas concretas de intervención, con la respectiva evaluación de impacto en el tiempo como lo sugieren Organización Mundial de la Salud (OMS, 2013) y el Ministerio de Salud (MINSA, 2014).

Como afirma Hernández (2014), Perú sigue entre los países de la región con menor productividad científica, tanto en relación con su población como con respecto de su riqueza. A pesar de la necesidad de investigaciones en la ciencia psicológica para dar respuesta a los problemas señalados, los estudios en esta área son insuficientes. Si se observa uno de los rankings mundiales de investigación más frecuentados, tal como es el propuesto por el SCImago Research Group (2016), se apreciará que no hay ninguna revista peruana de psicología con impacto científico.

Se observa, también, poco interés por desarrollar esta ciencia, pues como indica la UNESCO (2010), en muchos países, las Ciencias Naturales, en comparación a las Ciencias Sociales, siguen atrayendo la atención exclusiva en financiamiento. En Perú, los pocos incentivos económicos dados por instituciones del Estado a la investigación, como el Consejo Nacional de Ciencia, Tecnología e Innovación Tecnológica (CONCYTEC), se orientan 
más a las Ciencias Biológicas, Informáticas o Tecnológicas, en desmedro del avance de las Ciencias Sociales y de la Salud, como es la Psicología.

Según la UNESCO (2009), la Universidad es quien debería asumir el liderazgo social en materia de creación de conocimientos de alcance mundial a través de la investigación para abordar retos mundiales tal como es la salud pública. Por ello, las Facultades y/o Escuelas de Psicología, de las distintas universidades peruanas (públicas y privadas), deben contribuir con este objetivo incentivando la investigación en sus aulas, creando grupos de estudio, fomentando los estudios interdisciplinarios y sobre todo, promoviendo que sus egresados se titulen mediante la realización de una tesis, realidad que aún es muy lejana.

En este contexto cabe preguntarse ¿cuáles son los motivos o factores que influyen en la decisión de realizar o no realizar una tesis para obtener el título profesional en estudiantes de pregrado de la carrera de psicología? Al respecto, se encontraron algunos antecedentes en el extranjero aunque abordados en estudiantes de programas de maestría y/o doctorado en carreras distintas a la Psicología. Así, Ochoa (2011) encontró que las actividades como delimitar un tema, elaborar el estado de la cuestión y el marco teórico, hacer el diseño metodológico y redactar un informe de investigación son las actividades más complejas y, por tanto, son los momentos donde más se necesita de un acompañamiento. Se ha señalado también que factores personales como la actitud y la motivación (como aspectos cognitivos y metodológicos) son influyentes y necesarios para la realización de una investigación (Rietveldt \& Vera, 2012). El papel del docente como agente motivador, las asesorías recibidas de forma permanente y en general el apoyo de la institución también influyen en la realización de trabajos de investigación (Magaña, Aguilar, Pérez, Quijano \& Argüelles, 2014; Rietveldt \& Vera, 2012).

Otro grupo de estudios encontraron algunos factores de orden psicológico que influyen en actividades de investigación. Así, se ha encontrado evidencia sobre el papel mediador de la autoeficacia en este tipo de actividades (Alegre, 2014; Balloo, Pauli \& Worrell, 2016; Chumwichan, \& Siriparp, 
2016; Lambie, Hayes, Griffith, Limbert \& Mullen, 2013; Mohamed, \& Nordin, 2013; Reyes \& Gutiérrez, 2015); procesos motivacionales, sobre todo intrínsecos (Kozlova \& Atamanova, 2013; Rietveldt \& Vera, 2012); expectativas de los resultados de la investigación (Chumwichan \& Siriparp, 2016); actitudes hacia la investigación (Rietveldt \& Vera, 2012).

En Perú, estudios sobre el tema se realizaron con estudiantes de otras carreras profesionales diferentes a la Psicología, encontrándose aspectos que influyen en la realización de tesis como sensación de no ser capaz y no estar suficientemente preparado, deseo de contar con un marco teórico perfecto, miedo al trabajo de campo, el tipo de asesor que tienen, el reconocimiento social (de docentes y compañeros) y la actitud crítica de los profesores (Portocarrero \& Bielich, 2006). Por su parte, Alarco, Aguirre-Cuadros, Aliaga-Chávez y Álvarez-Andrade (2010), concluyeron que factores como la existencia de trámites engorrosos, los deficientes conocimientos en metodología de la investigación, la falta de recursos económicos y la falta de tiempo se asocian a la no realización de tesis.

Como puede observarse, en el contexto peruano son escasos los estudios empíricos realizados sobre esta problemática y ninguno abordó la realización de tesis en el campo de la Psicología. En congruencia, el siguiente estudio tiene como objetivo identificar, describir y explicar aquellos factores que influyen en la decisión de realizar o no realizar una tesis para obtener el título profesional en estudiantes de pregrado de la carrera de Psicología.

\section{Metodología}

\section{Enfoque de estudio y diseño.}

La investigación se inscribe dentro del paradigma de investigación constructivista. En ese sentido, el enfoque empleado es cualitativo. Se eligió esta metodología pues los objetivos de la investigación apuntan a identificar los motivos expresados por los mismos actores del proceso, en este caso, 
estudiantes de pregrado, tal y como ellos lo perciben. Interesa, por lo tanto, sus experiencias, sus opiniones y atribuciones que le dan al hecho de hacer o no un trabajo de investigación para obtener su título profesional.

Asimismo, el diseño o tradición elegido para estudiar este tema es la Teoría Fundamentada (Glaser \& Strauss, 1967; Strauss \& Corbin, 2002), dado que este método es adecuado para indagar y comprender procesos, interacciones entre constructos que influyen en otros, de tal forma que emergen de los datos teorías comprensivas sobre un tema específico tal como es la decisión de realizar o no realizar una tesis.

\section{Técnicas e instrumentos.}

Las técnicas empleadas fueron la entrevista cualitativa individual y grupos focales. Los instrumentos para recabar la información lo constituyen una guía de entrevista y guía de grupo focal. Los ejes temáticos de las guías respectivas fueron: modalidad para obtener el título profesional, importancia de realizar una tesis, razones para realizar una tesis, razones para no realizar una tesis. Para el registro de la información, se emplearon como herramientas un grabador de audio digital, equipos móviles con videograbadora, hojas de papel y lapiceros.

Respecto a la validez de las técnicas, es importante señalar que la entrevista cualitativa, como técnica de recolección de datos, tiene como objetivo asegurar que los participantes sean libres de cuestionar y, si es necesario, corregir las suposiciones del investigador sobre los significados investigados, plasmados en las preguntas de la guía de entrevista durante el mismo proceso de recolección de datos (Kvale, 2011). Asimismo, la información recolectada tuvo lugar en los entornos reales de los participantes, como son su centro de estudios y de práctica profesional, lo que significa que dichos estudios tienen una mayor validez ecológica (Willig, 2013). 


\section{Participantes.}

Se seleccionó a los colaboradores de acuerdo a criterios no probabilísticos, utilizando un muestreo intencional (Flick, 2015). Así, los participantes del estudio fueron en total 28 estudiantes de pregrado ( 20 mujeres y 8 varones), con edades comprendidas entre los 20 y 52 años, de diferentes niveles socioeconómicos, quienes actualmente residen en la ciudad de Lima y que se encuentran en los últimos ciclos de la carrea de Psicología de cinco universidades (dos instituciones nacionales y tres particulares). Del total de participantes, dos de ellos refirieron no haber elegido aún la modalidad de titulación, 9 de ellos manifestaron su interés por graduarse mediante una tesis y 17 expresaron su deseo de obtener su título profesional mediante otra modalidad, como curso o examen.

\section{Procedimiento.}

El proceso de recolección de datos se realizó de forma paulatina, entre los meses de noviembre de 2014 hasta abril de 2015, pues hubo algunos inconvenientes con la disposición de los integrantes del equipo de investigación.

Como primera actividad, el equipo de investigación realizó la guía de entrevista (individual y grupal) con los ejes temáticos relacionados al objetivo del estudio. Paralelamente, se elaboraron los consentimientos informados, las tarjetas de invitación y agradecimiento para los participantes. Cada integrante del equipo realizó las coordinaciones respectivas para acceder a los participantes. Para las entrevistas individuales se realizaron invitaciones de forma verbal a los estudiantes, ya sea en su contexto de trabajo (donde hacían sus prácticas profesionales) o en los ambientes de sus universidades. Para los grupos focales, se coordinó con los profesionales responsables del servicio psicológico de dos instituciones (un establecimiento de salud nacional y una clínica universitaria), donde los estudiantes realizaban su internado (prácticas pre profesionales acreditadas). Se establecieron el lugar, fecha y hora de las entrevistas. 
Las entrevistas individuales se realizaron en los ambientes de la universidad de los participantes, específicamente en las aulas de estudio. Los grupos focales (un grupo de 9 integrantes y otro de 12) se realizaron en el establecimiento donde los colaboradores realizaban su internado. Para esto, se utilizaron los salones que servían como centro de reuniones por los profesionales de esa área, previa coordinación con jefes del servicio de Psicología.

Se garantizaron las condiciones contextuales físicas adecuadas en los diferentes ambientes en que se recogió los datos. Se brindaron las mismas instrucciones y se contó con los materiales necesarios. De esta forma, en su mayoría, los/las informantes respondían a las preguntas con una actitud espontánea y colaboradora. Sólo en el primer grupo focal, una participante expresó su fastidio pues el responsable del servicio no le había informado el contenido de la reunión. A pesar de ello, al transcurrir la sesión, dicha colaboradora se mostró asequible y participó de manera activa, mostrando interés en el tema.

Una vez que se obtuvo la información de los participantes, se transcribieron las entrevistas a formato de texto Word, se editaron las mismas respetando en todo momento las expresiones originales de sus autores. Finalmente se procedió al procesamiento de datos.

\section{Procesamiento de datos.}

Es importante señalar que el procesamiento de datos cualitativos se basa en procesos y operaciones lógicas analíticas inductivas (Gibbs, 2012). En el estudio, se utilizó las estrategias sugeridas del método de la Grounded Theory (Glaser \& Strauss, 1967). De esta forma, la información obtenida fue segmentada en unidades de análisis (trozos o segmentos de datos) tomando como criterio de separación la unidad temática (segmento de texto que versa sobre un contenido o tema específico). Luego se realizó la categorización de primer nivel. Este proceso implica crear conceptos o categorías que 
representen el contenido del discurso expresado por los participantes a través de la comparación constantemente de las unidades de análisis. Se asignaron códigos a las categorías. Por tanto, en esta primera etapa del análisis, se obtuvo un primer sistema de categorías descriptivas que emergieron de las verbalizaciones de los participantes.

Luego de procesada así la información, se crearon categorías más analíticas e interpretativas mediante el proceso de categorización de segundo nivel, para lo cual se realizó la comparación contante de las categorías primarias (los conceptos obtenidos en el paso anterior), de tal forma que algunas de ellas se integraron en conceptos más abstractos y explicativos. Así por ejemplo, categorías como tiempo que demanda investigar, actividad laboral para satisfacer una necesidad económica y gasto económico para hacer tesis se agruparon en factores circunstanciales, pues todas ellos aluden a situaciones o eventos por los que atraviesa el estudiante durante su elección de realizar tesis o no realizarlo. Luego, se relacionaron entre sí las categorías finales para obtener patrones temáticos (categorías principales que tienen entre sí relaciones de causa- efecto, implicancia, coocurrencia, etc.), a fin de elaborar modelos comprensivos sobre el fenómeno estudiado mediante representaciones visuales. Se utilizó el programa informático Nvivo, versión 10 como ayuda para el manejo y organización de los datos.

\section{Criterios de calidad.}

A fin de garantizar la credibilidad y confirmabilidad del estudio, se registraron en audio las respuestas de los participantes, luego se transcribieron de tal manera que el texto reflejara el discurso de los participantes. Asimismo, se utilizó triangulación de técnicas de recolección de datos, tal como es la entrevista cualitativa individual y el grupo focal, lo cual puede contrastarse en la recuperación de unidades de análisis donde hay ejemplos de discursos individuales y del grupo focal. Se realizó triangulación de investigadores, de tal forma que los miembros del equipo de investigación analizaron los datos de forma individual y luego se compararon las categorías emergentes. Si bien 
hubo algunas diferencias en las categorías primarias (por ejemplo tiempo para hacer tesis, tiempo como causa o tiempo requerido para investigar), se coincidieron en los temas centrales (factores psicológicos, circunstanciales, relativos a la universidad, familiares y socioculturales).

La dependencia del estudio se logró mediante la elaboración de las guías de entrevista y grupos focales. Estas guías contenían los temas o preguntas principales del estudio y se aplicaron sin mayores modificaciones a los participantes. Otra estrategia empleada para este fin fue que el equipo de investigación aseguró que tanto el contexto y la forma como se aplicaron dichos instrumentos fueran similares.

La descripción detallada de las características de los colaboradores, el contexto de estudio y el procedimiento que se realizó al recolectar los datos, son algunas estrategias que el equipo de investigación consideró pertinente para optimizar la transferibilidad del estudio.

\section{Aspectos Éticos.}

El estudio realizado cumple con los aspectos éticos contemplados en los principios básicos de la American Psychological Association (2010) y el Código de Ética Profesional del Colegio de Psicólogos del Perú (s.f.). Así, los resultados obtenidos son producto de un proceso sistemático, crítico y empírico realizado por el equipo de investigación. Los aportes de otros investigadores pertinentes al desarrollo del siguiente estudio, han sido citados adecuadamente y figuran en la lista de referencias, evitando así la conducta antiética de plagio. La protección del bienestar y dignidad de las participantes se garantizó con el consentimiento informado verbal y/o escrito y la confidencialidad. Es conveniente mencionar que todos los participantes consintieron ser grabados en audio, de tal forma que la información recabada sea lo más fiel a sus aportes. 


\section{Resultados}

\section{Factores circunstanciales.}

Estos factores son definidos como los eventos o situaciones actuales que afrontan los participantes del estudio. El tiempo que demanda investigar, la actividad laboral (trabajo) que desempeñan o quieren desempeñar para satisfacer una necesidad económica fueron los motivos señalados que influyen en la decisión de hacer o no una tesis. Cabe señalar que estas categorías se encontraron tanto en las entrevistas individuales como en los dos grupos focales.

"Si tuviera tiempo disponible, no trabajaría, me gustaría hacer tesis" (S1).

"Para mi sería más factible por el mismo hecho que no tengo mucho tiempo, tengo que trabajar, costear mis gastos en la universidad” (S2) "El factor económico también. Empezar a trabajar de una vez, generar un ingreso..." (S3).

"...Lo he tenido pensado hacerlo por tesis pero creo que va a ser muy dificil, tanto porque trabajo y por el tiempo que dispongo" (Grupo focal 1).

"Yo concuerdo con los beneficios para otras personas. Yo inclusive he hecho dos trabajos cualitativos. Pero yo he optado más por el examen, por el tiempo, por cuestiones" (Grupo focal 2).

El gasto económico que implica realizar una investigación, también es señalado por los participantes como un factor que reduce la intención de hacer una tesis.

"Yo supongo que para hacer una buena investigación tendrías que contar con unos recursos económicos, para incluso viajar, o para hacer algunas investigaciones fuera" (S2).

"Otro punto también podría ser la inversión, porque para realizar un estudio de investigación, en este caso una tesis, aparte de la orientación, necesitas tener un presupuesto para poder realizarla" (Grupo focal 1). 
"Yo creo que sí [sobre el gasto económico], porque al momento que realizas encuestas tiene que sacar copias, tienes que ir a la biblioteca, implica un gasto" (Grupo focal 2).

\section{Factores psicológicos.}

Se definen como factores psicológicos aquel conjunto de componentes psicológicos cognitivos, afectivos, comportamentales o rasgos de personalidad que conforman al individuo, en este caso estudiante, y que facilitan $\mathrm{u}$ obstaculizan la realización de una investigación. Estos componentes, como procesos, se encuentran en constante interacción (Figura 1).

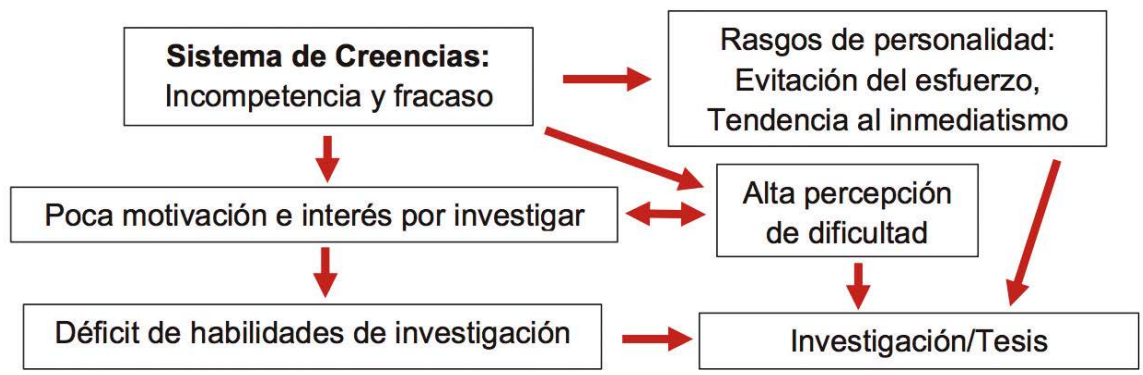

Figura 1. Influencia de factores psicológicos en la decisión de realizar o no realizar investigación/tesis.

Un primer componente lo constituye el sistema de creencias. Este sistema se construye a lo largo de la vida de la persona y es producto de la interacción de muchos factores. En el contexto del estudio, el contenido que adopte este sistema sobre uno mismo o sobre la actividad de investigar influye en la decisión de hacer o no hacer tesis. Si hay contenidos poco reales, es probable que se opte por otras opciones y no se investigue.

"Todas las semanas la profesora me decía que tengo que revisarla [curso de trabajo de investigación], y no sé, como que he sentido que no lo estoy haciendo bien e imagínate si tengo que hacer para el título y me dicen que no pasa o algo así; no me imagino otro año estando 
preparando otra tesis, me parece un poco atemorizante más que todo" (S4).

"Me imagino que si haces la tesis, y qué pasa cuando la presentas y tienes miedo al rechazo que tu tesis no ha sido aceptada" (S1).

“...y si no me sale bien, si me la rechazan, si no tengo un asesor; yo no sé, nunca he aprendido" (S5).

Los rasgos de personalidad evitación del esfuerzo (tendencia a evadir actividades que impliquen esfuerzos cognitivos) y tendencia al inmediatismo (querer obtener o conseguir metas de forma inmediata), son patrones que se hallaron en el estudio.

"...Por lo mismo que han dicho mis compañeros, el tiempo. Quiero sacar rápido el bachiller y ya sacar mi título, rápido" (Grupo focal 1).

"Yo quiero cuanto antes mi titulación para poder trabajar, y eso lo puedo tener en solo 6 meses" (Grupo focal 2).

Asimismo, la percepción de dificultad que presenta el estudiante respecto a investigar, condicionará su decisión de hacer o no tesis.

"O también algunos dicen que es muy dificil y prefieren irse por las cosas más sencillas, dicen 'ni importa, gasto más pero la tesis no '”' (S5).

"Me parece importante porque es una investigación, pero es un poco tedioso por lo mismo que te va a llevar a recabar información, investigación, datos, y lo veo muy complicado para mí" (S1).

“Actualmente, desde mi posición, sería más factible para mí obtener el título llevando un curso de actualización. Por experiencias lo veo un poco más sencillo" (S2). 


\section{Factor familiar.}

La figura 2 muestra los factores familiares que surgieron del análisis. Así, las estrategias que la familia utilizó para criar a sus hijos, los patrones de crianza, particularmente las relacionadas con el proceso de aprendizaje, así como el apoyo familiar, entendido como la ayuda económica y/o moral, el costear los gastos de educación y alentar anímicamente a que se continúe con los estudios durante el proceso de aprendizaje académico, constituyen los dos factores familiares que influyen en la realización o no de un trabajo de investigación.

“...Y desde mis recuerdos, no tenía muchos límites en casa, de manera que si tenía la oportunidad de desarmar un reloj despertador, un teléfono, lo hacía, lo exploraba y lo destruía, pero me daba esa satisfacción de investigar, y no había ese grito de 'qué has hecho'” (Grupo focal 2).

"Considero que es importante el apoyo de mis padres...Mis padres me apoyan económicamente, también anímicamente. Un soporte familiar pienso que también es importante" (Grupo focal 1).

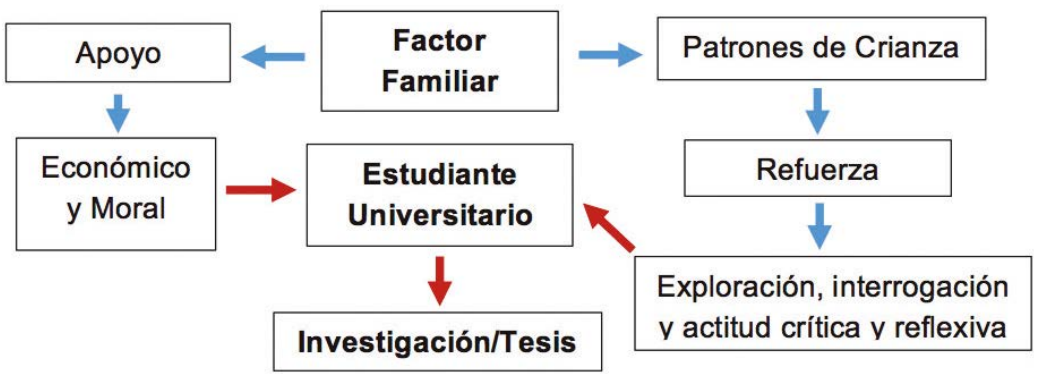

Figura 2. Factores familiares que influyen en la decisión de realizar o no realizar investigación/tesis.

\section{Factores relativos a la universidad.}

Este factor hace referencia al apoyo por parte de la Institución Educativa Superior o Universidad, representada por las autoridades de las Facultades y/o Escuelas de Psicología, que, a través de sus docentes, procesos y estrategias, 
favorecen u obstaculizan la investigación en sus estudiantes y la realización de una tesis para obtener el título profesional (Figura 3).

Se encontró que el plan curricular que posee la Universidad, tanto en su estructura y contenido que incluyan actividades de investigación y el cumplimiento de dicho plan, influyen en la realización de estudios científicos por parte de los estudiantes.

"[Refiriéndose a algunas universidades]...Están limitadas en el aprendizaje, el conocimiento, la investigación. Solo hay enseñanzas básicas" (S1).

"Yo creo que sería muy importante que ciertas universidades modifiquen su maya curricular ...En cambio, en mi universidad solamente teníamos tres cursos [de investigación], si no me equivoco. Entonces, digamos que ellas [compañeras de otra universidad] tienen más oportunidades y más capacidades para poder hacer una tesis en comparación con otras universidades" (Grupo focal 1).

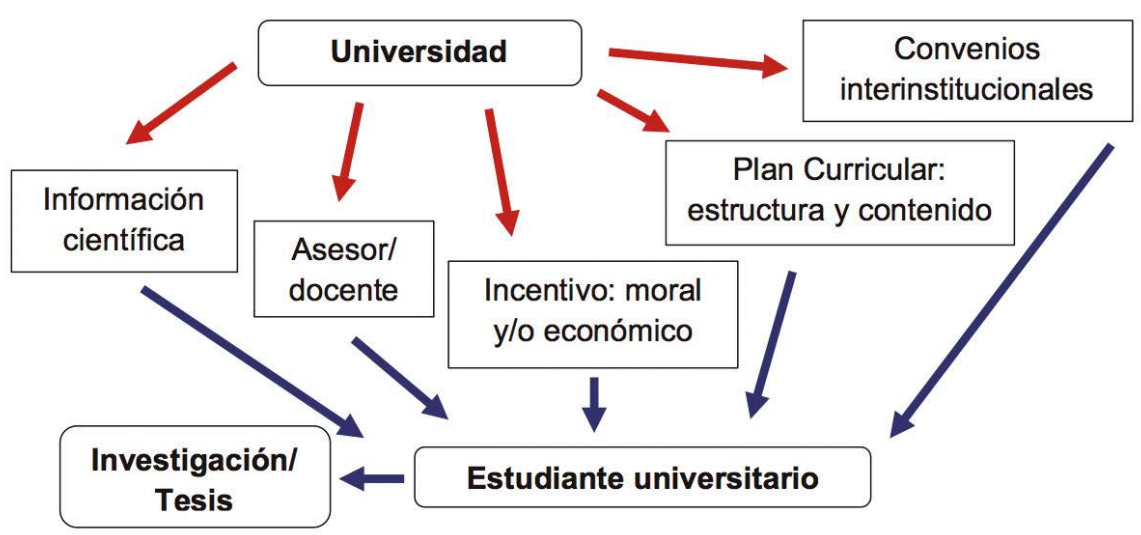

En esa misma línea, los convenios interinstitucionales que realiza la Universidad con entidades como empresas, establecimientos de salud, instituciones educativas, etc., representan para los estudiantes un apoyo importante para hacer una tesis. 
"Yo me inclino hacia el área organizacional..., y en estos momentos no cuento con prácticas que me puedan mostrar cómo es el área organizacional y qué problema es un problema palpable que yo tenga cerca. Como no tengo eso, se me hace dificultoso pensar en una tesis en estos momentos" (S4).

“...En realidad no había un tema que realmente me llamara la atención, que me impulsara...Me preguntaba por qué pasaba esto. En mi caso, había sido porque la mayoría, al menos los de mi profesión, habían tenido el contacto, prácticas por medio de voluntariados, y de esa forma había despertado el interés...En mi caso no fue así, es la primera vez que estoy teniendo contacto con la práctica, con las personas, y creo que es aqui donde voy a poder encontrar realmente un tema que sí sea de mi interés" (Grupo focal 2).

El incentivo económico y moral que propicia la Universidad también emergió como un aspecto importante en el proceso de análisis. Los/las participantes perciben que esta motivación externa por parte de la Universidad contribuye a que sus alumnos se inclinen por actividades de investigación. Además, se encontró que si bien hay un incentivo moral por parte de las autoridades y docentes para hacer una tesis, es escaso o nulo un apoyo o incentivo económico en esta área.

"Además, no se motiva mucho; siempre se habla que hay que hacer tesis pero no se da el apoyo a los estudiantes" (S6).

"Me he dado cuenta que la universidad no nos apoya en nada, más que en cartas de presentación, un apoyo económico de la universidad ayudará bastante a la investigación” (S1).

"Conversando con mis compañeros, no he escuchado uno que diga que sus investigaciones les apoye la universidad. Yo, particularmente, no he escuchado eso..." (Grupo focal 1).

La información cientifica que posee la Universidad, expresado en bibliotecas adecuadamente equipadas, producciones científicas (tesis o 
artículos) que se plasmen en revistas científicas propias y adscripciones a bases de datos internacionales con información pertinente, representa para el estudiante un apoyo más.

"Yo para hacer las últimas investigaciones o trabajos que hice, yo no recurri a la biblioteca, porque cuando pedi un diccionario, tenía un diccionario sólo psicoanalítico; pedí otras cosas y tenían cosas muy básicas y limitadas; luego yo ya no busqué más" (S5).

El asesor/docente, como representante directo de la Universidad, es identificado como un factor importante para los participantes. Se halló que la didáctica de éste, el dominio de tema que pueda tener, su estilo de enseñanza, la experiencia en investigación y el interés por enseñar a investigar, son características deseables por los estudiantes.

"Creo que hay profesores muy buenos que saben demasiado, tienen buenos conocimientos, pero lastimosamente no saben cómo expresarlo, cómo ayudar, ese es el problema que hay. Me ha pasado” (S7).

"Para mí tiene que saber bastante sobre el tema" (S3).

"No sé si acá habrá, pero en otros lugares sí hay asesores que no hacen investigación, simplemente son asesores" (S5).

"Bueno en mi universidad, ha habido problemas con el asesor porque no tiene tiempo, hay que ir a buscarlo, molestarlo..., incluso hasta ir a su casa...Algunos no toman interés" (Grupo focal 1).

\section{Factores socioculturales.}

Los factores socioculturales hacen referencia al conjunto de entidades, políticas, situaciones y/o representaciones sociales relacionadas con la investigación y que influyen en la decisión de realizar una tesis. En este sentido, se identifica el sistema de educación básica regular como insuficiente $o$ deficitario para incentivar la investigación desde temprana edad, pues 
sigue un modelo educativo tradicional que no favorece el desarrollo del pensamiento crítico y la indagación.

"A los niños se les debe incentivar desde temprana edad, desde el curso de ciencia, biología; darles esa motivación para que ellos mismos busquen, aumentar esa curiosidad que se despierta a temprana edad" (Grupo focal 1).

"[Sobre el pensamiento crítico]...Yo creo que eso va desde antes de la universidad, desde el colegio. A veces los profesores vienen, dictan clase, tal fecha es el examen y punto. En el estatal no es como que te ahondan más a buscar ese sentido crítico para que puedas seguir haciéndola en la etapa universitaria. Porque yo he visto que, en los primeros siglos de la universidad, es allí donde le choca o le cuesta al alumno adaptarse" (Grupo focal 2).

También se señala que los cambios en las políticas de educación superior que el Estado realizó hace algunos años atrás, fueron desfavorables para la realización de tesis con el fin de obtener el título profesional.

"Yo tuve la oportunidad de estar en la universidad hace ya bastantes años, antes de que se diera un poco ese vacio que generó el bachillerato automático y todo automático; entonces el valor que se le daba al trabajo de investigación antes era algo natural, tácito, implícito en la carrera” (Grupo focal 2).

Asimismo, los participantes perciben que la sociedad y cultura de la que forman parte, como entidad construida, no valora el desarrollo de investigación y no hay ventajas comparativas para quien obtiene su título profesional mediante una tesis.

"Entonces hacer una investigación te da un poco más de nivel, pero el título en sí es el mismo, no cambia” (S2).

"En el campo laboral, no tiene peso [el hacer tesis]" (Grupo focal 2).

"No tenemos la cultura de investigar, y eso se ha dado desde antes" (S6). 


\section{Discusión}

El propósito del siguiente estudio fue identificar, describir y explicar aquellos factores que influyen en la decisión del estudiante universitario para realizar o no realizar una tesis de grado. Los resultados sugieren que existen factores psicológicos, circunstanciales, familiares, relacionados a la universidad, sociales y culturales cuya compleja interacción influye en el proceso evaluativo cognitivo y reflexivo del estudiante para tomar esta decisión, representando el sistema de creencias del estudiante respecto de sí mismo y en relación con la actividad de investigación un factor psicológico central.

Los factores circunstanciales, tales como el tiempo, necesidad económica (Alarco et al., 2010), querer trabajar y los gastos que implica investigar, son factores advertidos por los participantes sin mayor esfuerzo y se presentan como los más apremiantes en el proceso de evaluación para tomar una decisión y optar por no investigar (CONCYTEC, 2014). Si bien estos factores explicarían la decisión tomada, confluyen otros factores que requieren de un mayor esfuerzo para reconocerlos como tales. Son aquellos procesos que se han dado en su desarrollo psicosocial y que aún están vigentes. Estos procesos se han automatizado a tal punto que difícilmente se toma conciencia de sus efectos en la decisión de hacer o no investigación y adquieren un rol fundamental en la evaluación.

Así, existen factores psicológicos que influyen directamente en la decisión de realizar tesis. De estos factores, el sistema de creencias que un estudiante tiene respecto de sí mismo (en este caso, en el contexto académico), de sus habilidades en relación con la investigación y las ideas que tiene sobre la actividad de investigar, se convierten en un factor central y es el punto medular en el proceso de evaluación que realiza. Se puede observar algunas aproximaciones al respecto en Portocarrero y Bielich (2006) y en estudios sobre autoeficacia (Alegre, 2014; Balloo et al, 2016; Chumwichan, 
\& Siriparp, 2016; Lambie et al 2013; Mohamed, \& Nordin, 2013; Reyes \& Gutiérrez, 2015).

Las creencias con contenidos poco racionales promueven el desarrollo de rasgos de personalidad poco adaptativos (Dryden, David \& Ellis, 2010), como el rasgo evitación del esfuerzo y tendencia al inmediatismo. Así, posiblemente se optará por alternativas o soluciones más fáciles para evitar que se cumplan dichas creencias y el consecuente malestar emocional. A su vez, dichas creencias sesgarán la percepción de dificultad sobre la actividad de investigación y se concluirá que "hacer una tesis es muy difícil" o es "complicado". El autoconcepto que el estudiante tenga de sí mismo producto de su sistema de creencias, influye en su motivación académica (Carranza \& Apaza, 2015), en este caso, motivación e interés por investigar, siendo aspectos relevantes para hacer una investigación (Kozlova, \& Atamanova, 2013; Rietveldt y Vera, 2012), lo que genera que el estudiante no desarrolle habilidades de investigación, como por ejemplo revisar literatura, delimitar un tema o realizar el diseño metodológico (Ochoa, 2011). Todo ello repercutirá en la decisión de investigar o no hacerlo.

Ahora bien, los estudiantes construyeron dicho sistema durante su proceso de socialización, y lo sigue haciendo en la medida que interactúa con los diferentes agentes socializantes. Por ello, este sistema es dinámico y sus contenidos son susceptibles de modificarse o perpetuarse.

La familia, como primer grupo social, es el primer agente socializador que influirá en el proceso de construcción del sistema de creencias por parte del alumnado, con la consecuente limitación de su ámbito de exploración, su ímpetu curioso y su disposición natural de investigar. Madre y padre, por priorizar patrones de crianza orientadas a la calma momentánea o simplemente porque se reproducen mensajes automáticos que ellos mismos recibieron, transmiten mensajes con carga emocional que prohíben la exploración del medio, dejando sin respuesta las preguntas que los hijos se van planteando sobre su entorno. Ello genera pensamientos de inseguridad, temor ante lo nuevo, disminución de la actividad conductual, desinterés por responder sus 
propias preguntas y con ello, disminución de formularse preguntas sobre lo desconocido. La importancia de orientar a los padres sobre las consecuencias de este tipo de pautas de crianza se hace necesario por los efectos que tendrá en el desarrollo de sus competencias no solo académicas, sino también emocionales y sociales.

El segundo agente socializante, la escuela básica regular, también contribuye con el aplacamiento del ímpetu investigador del ser humano a través del fomento de contenidos poco racionales de pensamiento sobre investigación. El sistema educativo actual aún tiene sistemas de enseñanzaaprendizaje tradicionales y desactualizados, donde es el docente quien tiene el conocimiento y no se debe cuestionar la información que propicia. En este contexto, el aprendizaje que los educandos adquieran estará sesgado por la visión de aquellos. No se construye el aprendizaje, solo se replica. Y no solo en muchas de las instituciones del Estado pasa ello, sino también en los colegios particulares y religiosos.

Es necesario, entonces, que se desarrolle un sistema de educación básica regular de calidad, pues tendrá el potencial de desarrollar en los estudiantes, en primera instancia, y como componente medular, un sistema de creencias con contenidos racionales sobre el aprendizaje y la investigación; y, sobre la base de dichas creencias, promoverá habilidades académicas y socioemocionales adecuadas. Una de esas habilidades, el pensamiento crítico, adquiere particular relevancia (López, 2012). Si el docente incentiva esta destreza aumentará la posibilidad de que el estudiante investigue, pues querrá comprobar cuanto se le dice, buscará información en diferentes fuentes, elaborará supuestos y construirá sistemas explicativos sobre un fenómeno. De esta forma, se abre el camino para prepararlos para la educación superior, para la producción científica. No podrán ser igualmente logradas dichas habilidades en la educación superior si no fueron iniciadas a la edad apropiada en la educación básica regular (Sistema Nacional de Evaluación, Acreditación y Certificación de la Calidad Educativa [SINEACE], 2013; Segrera, 2016). 
El panorama educativo discutido anteriormente no es muy distinto en las Universidades, puesto que en muchas de ellas aún se aplican modelos verticales y autoritarios de enseñanza, sistemas de aprendizajes obsoletos, expositivos y poco innovadores (Hernández y Alberto, 2012), cuya finalidad muchas veces es adiestrar a sus estudiantes en determinadas formas de pensar y actuar. El juicio crítico en esas condiciones no se desarrolla pues se cree y se aprende a no pensar de forma independiente, a no innovar y no investigar. Se afianzan viejos patrones y contenidos de pensamiento en torno a la investigación, y, como consecuencia final, se optará por no realizar estudios pues se cree que todo ya está dado y no se puede cambiar la realidad psicosocial.

En esta misma línea de análisis, la situación o el contexto relacionado con la información académica y científica que brinde la universidad, influye también en el sistema de creencias del estudiante y, esto a su vez, condiciona la percepción de dificultad que se tiene para hacer una investigación. Cuando el estudiante no encuentra los recursos disponibles en la universidad, además que los facilitadores (docentes) no le han enseñado a buscarlos, se refuerzan contenidos de pensamientos irracionales, como "hacer tesis es difícil". Entonces, si la Universidad no invierte en recursos para la investigación, no puede esperar que sus alumnos se gradúen realizando un trabajo de investigación. En este sentido, los escasos textos producidos de manera científica, como efecto de ese no interés, repercuten también en la percepción de dificultad. Al encontrar pocos modelos de personas que han realizado y publicado investigaciones, se cree que poco posible.

Asimismo, en la medida que las Universidades puedan crear convenios con instituciones serias y pertinentes en el desarrollo de la carrera de psicología (en sus áreas clínica, psicológica, organizacional, comunitaria, etc.), los estudiantes podrán tener mayor contacto con la realidad, percibirán de cerca los distintos problemas o temas que son necesarios abordarlos, estudiarlos e investigarlos. De esa manera, se dispone de una gama de temas potenciales para investigar, de los cuales pueden derivarse alternativas 
de solución y eventualmente crear programas o instrumentos nuevos que puedan ser patentizados por psicólogos peruanos. De lo contrario, es posible que los estudiantes carezcan de ideas relevantes para su estudio, y de realizarse alguna investigación, los resultados de ésta no responderán a las necesidades del país (CONCYTEC, 2016), aumentando la brecha entre la práctica profesional y la producción científica que se observa en la Psicología peruana. En concordancia con lo que manifiesta la UNESCO (2009), son las universidades las que deberían buscar áreas de investigación y docencia para abordar aquellos temas relevantes para el bienestar de la población y, sobre ello, crear bases afirmadas para la ciencia y la tecnología pertinentes en el ámbito local.

Un aspecto trascendental en este contexto es el papel del asesor/docente (Portocarrero y Bielich, 2006; Magaña et al. 2014), pues es quien, en primera instancia, representa a la Universidad y es quien tiene una relación directa y cercana con el estudiante. Como asesor y mediante sus prácticas y orientaciones, puede detectar y modificar el contenido del sistema de creencias del estudiante respecto a su competencia como investigador/a. Para esto, el asesor debe de actualizarse constantemente no sólo en contenido teórico sino también en estrategias didácticas y colaborativas para trasmitir y entrenar a sus estudiantes (Sayós, Pagés, Amador y Jorba, 2014; Abadía et al., 2015), de tal forma que transforme la clase en una comunidad de enseñanza-aprendizaje (Roselli, 2016). Más importante aún, el asesor debe realizar investigaciones, pues solo en la práctica podrá identificar aquellos temas centrales del proceso que serán necesarios para una enseñanza óptima (García-Gallego, Georgantzís, Martín-Montaner \& Pérez-Amaral, 2015).

En relación también con la Universidad, su plana docente y personal administrativos deben orientar adecuadamente a los estudiantes respecto a cómo realizar una tesis. Otras estrategias pueden ser reducir, agilizar y/o simplificar los trámites administrativos implicados, así como incentivar constantemente la elección de esta modalidad de titulación, de tal forma que los mitos o las ideas de los estudiantes respecto a la tesis ("es tedioso", 
"es complicado", "requiere tiempo", etc.) se contrasten con la realidad y se modifiquen.

Por otro lado, los factores socioculturales tienen la propiedad de ser el contexto raíz que sustenta los demás factores identificados que influyen en el sistema de creencias del estudiante respecto a sí mismo/a en relación a la investigación, y por ende, en la decisión de realizar una tesis. Como se mencionó al principio de esta discusión, estos factores son difícilmente identificables, pues parece que son muy lejanos a la realidad de los estudiantes.

Las políticas y normas educativas improvisadas, un modelo educativo tradicional e insuficientes políticas de incentivo a la investigación, en general, y la investigación psicológica, en particular, fomenta una educación básica regular deficitaria, un sistema universitario de insuficiente calidad, algunas orientadas por fines distintos a la formación, donde la calidad y la investigación pasan a un segundo plano y se opta por estrategias facilistas para generar profesionales egresados, lo que puede derivar en una sociedad que no investiga y una cultura que no tiene interés en la ciencia. Estas condiciones tienen el potencial de influir en el sistema de creencias del estudiante universitario y, por tanto, en la actividad de investigación.

Como consecuencia, la investigación científica en psicología será limitada, pues parece que poco interesa este tipo de estudios. Ya en el estudio realizado por CONCYTEC (2014) se observaba que las áreas de ciencias de la Salud y Psicología son disciplinas donde hay menos estudiantes interesados en realizar sus trabajos de investigación. Parece ser que es más importante invertir en actividades relacionadas a la economía de subsistencia, orientadas a la exportación de materias primas (Maguiña-Vargas, 2013) que en actividades de investigación para mejorar las condiciones de salud psicológica y social.

Como puede advertirse a lo largo de la discusión, el tránsito que se hace desde factores circunstanciales y psicológicos que afectan de forma directa a los participantes, hacia los sociales y culturales que operan más 
bien como condicionantes, evidencian el carácter complejo y dinámico de este problema, pues implica la interacción entre sistemas. De forma similar, la UNESCO (2010) identifica tres niveles de capacidad en el proceso de investigación: individual, de organización y sistémico, concluyendo que "si se quiere desarrollar la capacidad de investigación es necesario que los gobiernos, las organizaciones internacionales y los organismos de ayuda proporcionen financiación para las instituciones de investigación, así como formación individual (p. 22)”.

Los autores del estudio consideran que los cambios producidos por la reforma educativa superior en Perú, en el campo de la investigación, ordenados bajo la Nueva Ley Universitaria (Ley $N^{\circ} 30220$, 2014), no garantizan una mejora o desarrollo de la investigación por sí misma, pues más bien se puede interpretar como exigencias a las Universidades para tener profesionales graduados mediante trabajos de investigación. Si esa consigna se da de forma aislada, sin los cambios que son necesarios en los otros factores implicados, como se señaló en el presente estudio, es posible que se incurra en conductas antiéticas de investigación, como mandar a hacer una tesis a personas u organismos cuyo fin es lucrativo, o que las facultades de psicología de las diferentes universidades de Perú bajen sus estándares de calidad para aprobar una tesis.

Por supuesto, es importante señalar que los resultados del estudio representan solo las experiencias de los participantes, y, en ese sentido, no se conocen las opiniones y vivencias de estudiantes de otras universidades y regiones, o de otros actores de la comunidad universitaria. A pesar de ello, los hallazgos obtenidos constituyen un aporte para la comprensión del problema estudiado en la medida que es una aproximación explicativa creada a partir de los datos. En este sentido, se sugiere contrastar los modelos obtenidos, ampliarlos o mejorarlos en estudios que contemplen diferentes estudiantes de pregrado de Psicología, en diferentes contextos universitarios, ya sean nacionales o particulares, de Lima o de provincia, a fin de poder identificar y comprender la interacción de aquellos factores que influyen en la decisión 
del estudiante para investigar, y, en consecuencia, para decidir realizar o no una tesis.

\section{Referencias}

Abadía, A., Bueno, C., Ubieto-Artur, M., Márquez, M., Díaz, S., Jorba, H., \& Pagès, T. (2015). Competencias del buen docente universitario. Opinión de los estudiantes. REDU. Revista de Docencia Universitaria, 13(2), 363-390. Doi: https://doi.org/10.4995/redu.2015.5453

Alarco, J., Aguirre-Cuadros, E., Aliaga-Chávez, Y., \& Álvarez-Andrade, E. (2010). Factores asociados a la realización de tesis en pregrado de Medicina en una universidad pública del Perú. CIMEL, 15(2), 66-70.

Alegre, A. (2014). Autoeficacia académica, autorregulación del aprendizaje y rendimiento académico en estudiantes universitarios iniciales. Propósitos y Representaciones, 2(1), 79-120. Doi: https://doi.org/10.20511/pyr2014. $\mathrm{v} 2 \mathrm{n} 1.54$

Arias, F. (2006). Mitos y errores en la elaboración de tesis y proyectos de investigación. Caracas: Editorial Episteme. American Psychological Association. (2010). American Psychological Association ethical principles of psychologists and code of conduct. Recuperado de: http:// www.apa.org/ethics/code/index.aspx

Balloo, K., Pauli, R., \& Worrell, M. (2016). Individual differences in psychology undergraduates' development of research methods knowledge and skills. Procedia-Social and Behavioral Sciences, 217, 790-800. Doi: https://doi.org/10.1016/j.sbspro.2016.02.147

Carranza, R., \& Apaza, E. (2015). Autoconcepto académico y motivación académica en jóvenes talento de una universidad privada de Tarapoto. Propósitos y Representaciones, 3(1), 233-263. Doi: https://doi. org/10.20511/pyr2015.v3n1.72

Chumwichan, S., \& Siriparp, T. (2016). Influence of research training environment on research interest in graduate students. Procedia-Social and Behavioral Sciences, 217, 950-957. Doi: https://doi.org/10.1016/j. sbspro.2016.02.065 
Colegio de Psicólogos del Perú (s.f.). Código de Ética Profesional. Recuperado de http://www.colegiodepsicologosperu.org/w/imagenes/ enlaces/archivos/CodigoEticaPeru.pdf.

Consejo Nacional de Ciencia, Tecnología e Innovación Tecnológica CONCYTEC (2014). Situación de la formación de capital humano $e$ investigación en las universidades peruanas. II Censo Nacional Universitario 2010. Lima: Autor.

Consejo Nacional de Ciencia, Tecnología e Innovación Tecnológica CONCYTEC (2016). Política nacional para el desarrollo de la ciencia, tecnología e innovación tecnológica - CTI. Lima: Autor.

Defensoría del Pueblo (2008). Informe Defensorial $N^{o}$ 140. Salud Mental y Derechos Humanos. Supervisión de la política pública, la calidad de los servicios y la atención a poblaciones vulnerables. Lima: Autor.

Dryden, W., David, D., \& Ellis, A. (2010). Rational emotive behavior therapy. In K.S. Dobson (Ed.), Handbook of cognitive-behavioral therapies (3 ${ }^{\mathrm{a}}$ ed.) (pp. 226-276). New York: The Guilford Press.

Flick, U. (2015). El diseño de investigación cualitativa. Madrid: Ediciones Morata.

García-Gallego, A., Georgantzís, N., Martín-Montaner, J., \& Pérez-Amaral, T. (2015). (How) Do research and administrative duties affect university professors' teaching? Applied Economics, 47(45) 4868-4883. Doi: https:// doi.org/10.1080/00036846.2015.1037438

Gibbs, G. (2012). El análisis de datos cualitativos en Investigación Cualitativa. Madrid: Ediciones Morata.

Glaser, B.G., \& Strauss, A.L. (1967). The Discovery of Grounded Theory: Strategies for Qualitative Research. New York: Aldine.

Hernández, R. (2014) ¿Quién escribe más y sobre qué? Cambios recientes en la geopolítica de la producción científica en América Latina y el Caribe. Lima: Instituto de Estudios Peruanos.

Hernández, I., \& Alberto, J. (2012). El docente investigador frente al desarrollo humano. Revista Universitaria: Docencia, Investigación e Innovación, 1, 1-21. 
Instituto Nacional de Estadística e Informática (2011). II Censo Nacional Universitario 2010. Primeros resultados. Lima: Autor.

Kozlova, N. V., \& Atamanova, I. V. (2013). The Development of Undergraduates Motivation for Research Work. Procedia-Social and Behavioral Sciences, 93, 498-502. doi: https://doi.org/10.1016/j. sbspro.2013.09.227

Kvale, S. (2011). Las entrevistas en investigación cualitativa. Madrid: Ediciones Morata.

Lambie, G., Hayes, B., Griffith, C., Limbert, D., \& Mullen, P. (2013). An exploratory investigation of the research self-efficacy, interest in research, and research knowledge of Ph.D. in education students. Innovative Higher Education, 39 (2), 139-153. Doi: https://doi.org/10.1007/s10755013-9264-1

Ley No 30220, Ley Universitaria. Diario Oficial El Peruano, Lima, Perú, 09 de julio de 2014.

López, G. (2012). Pensamiento crítico en el aula. Docencia e Investigación, 22, 41-60.

Maguiña-Vargas, C. (2013) ¿Por qué investigar en el Perú? Revista del Cuerpo Médico del Hospital Nacional Almanzor Aguinaga Asenjo, 6(3), 6-8.

Magaña, D. E., Aguilar, N., Pérez, M., Quijano, R.A., \& Argüelles, L.A. (2014). Motivaciones y limitantes en la formación en investigación a través del programa de verano científico: un estudio en una muestra de estudiantes universitarios. Revista internacional administracion \& finanzas, 7(6), 103-120.

Ministerio de Salud. Dirección General de Epidemiología (2014). Carga de enfermedad en el Perú. Estimación de los años de vida saludables perdidos 2012. Lima: Autor.

Mohamed, F., \& Nordin, R. (2013). Research Efficacy among Engineering and Science Undergraduates. Procedia-Social and Behavioral Sciences, 102, 164-168. Doi: https://doi.org/10.1016/j.sbspro.2013.10.729 
Montemayor-Borsinger, A. (2005). La tesis. En Cubo, L. (Ed.). Los textos de la ciencia. Principales clases de discurso académico-científico (pp. 267-284). Córdoba: Ed. Comunicarte.

Ochoa, L. (2011). La elaboración de una tesis de maestría: exigencias y dificultades percibidas por sus protagonistas. Entornos, 24, 171-183.

Organización Mundial de la Salud (2013). Plan de acción sobre salud mental 2013-2020. Ginebra: Autor.

Portocarrero, F. (2014). "Bestias frágiles aunque tenaces": el incierto futuro de la educación superior en el Perú. En Blanco \& Negro, 5(2). Recuperado de: http://revistas.pucp.edu.pe/index.php/enblancoynegro/ article/view/11386/11899.

Portocarrero, G., \& Bielich, C. (2006) ¿Por qué los estudiantes no hacen sus tesis? Centro de Investigaciones Sociológicas, Económicas, Políticas y Antropológicas (CISEPA). Recuperado de: http://testdepartamento. pucp.edu.pe/comunicaciones/images/documentos/cap02 gportocarrero. pdf

Reyes, M.R., \& Gutiérrez, J. (2015). Sentido de autoeficacia en investigación de estudiantes de posgrado. Sinéctica, 45. Recuperado de: http://www. scielo.org.mx/pdf/sine/n45/n45a11.pdf.

Rietveldt, F., \& Vera, L. (2012). Factores que influyen en el proceso de elaboración de la tesis de grado. Omnia, 18(2), 109-122.

Roselli, N. (2016). El aprendizaje colaborativo: Bases teóricas y estrategias aplicables en la enseñanza universitaria. Propósitos y Representaciones, 4(1), 219-280. Doi: https://doi.org/10.20511/pyr2016.v4n1.90

Sabino, C. (1998). Cómo hacer una tesis. Y elaborar todo tipo de escritos. Buenos Aires: Lumen-Humanitas.

Sayós, R., Pagés, T., Amador, J., \& Jorba, H. (2014). Ser buen docente ¿Qué opinan los estudiantes de la universidad de Barcelona? Revista Iberoamericana de Psicología y Salud, 5 (2), 135-149.

SCImago Research Group (2016). SCImago Journal and Country Rank [Archivo de datos]. Recuperado de http://www.scimagojr.com/ countryrank.php? area $=3200 \&$ category $=0 \&$ region $=$ Latin + America $\& y e a$ $\mathrm{r}=2014 \&$ order $=$ it\&min $=0 \& \min \_$type $=$it 
Segrera, F. L. (2016). Educación superior comparada: tendencias mundiales y de América Latina y Caribe. Avaliação: Revista da Avaliação da Educação Superior, 21 (1), 13-32. Doi: https://doi.org/10.1590/S141440772016000100002

Sistema Nacional de Evaluación, Acreditación y Certificación de la Calidad Educativa- SINEACE (2013). Educación superior en el Perú: retos para el aseguramiento de la calidad. Lima: Autor.

Strauss, A., \& Corbin, J. (2002). Bases de la investigación cualitativa. Técnicas y procedimientos para desarrollar teoría fundamentada. Colombia: Universidad de Antioquia.

UNESCO (2009). Conferencia Mundial sobre la Educación Superior - 2009: la nueva dinámica de la educación superior y la investigación para el cambio social y el desarrollo. Paris: Autor.

UNESCO (2010). El Informe mundial sobre las ciencias sociales 2010. Divisorias del conocimiento. Resumen. Paris: Autor.

Willig, C. (2013). Introducing qualitative research in psychology ( $\left.3^{\mathrm{a}} \mathrm{ed}.\right)$. UK: McGraw-Hill Education. 